\begin{tabular}{|c|l|}
\hline Title & $\begin{array}{l}\text { Enhancement in selective mitochondrial association by direct modification of a mitochondrial targeting signal peptide } \\
\text { on aliposomal based nanocarrier }\end{array}$ \\
\hline Author(s) & Y amada, Y uma; Harashima, Hideyoshi \\
\hline Citation & $\begin{array}{l}\text { Mitochondrion, 13(5), 526-532 } \\
\text { https:/doi.org/10.1016/.mito.2012.09.001 }\end{array}$ \\
\hline Issue Date & 2013_09 \\
\hline Doc URL & http://hdl.handle.net/2115/54782 \\
\hline Type & article(author version) \\
\hline File Information & Wos_62771_Yamada_Harashima.pdf \\
\hline
\end{tabular}

Instructions for use 


\title{
Enhancement in selective mitochondrial association by direct modification of a mitochondrial targeting signal peptide on a liposomal based nanocarrier
}

\author{
Yuma Yamada, ${ }^{\dagger}$ Hideyoshi Harashima, ${ }^{*},{ }^{\dagger}$ \\ †Laboratory for Molecular Design of Pharmaceutics, Faculty of Pharmaceutical Sciences, Hokkaido \\ University, Kita-12, Nishi-6, Kita-ku, Sapporo 060-0812, Japan,
}

*To whom correspondence should be addressed: Laboratory for Molecular Design of Pharmaceutics,

Faculty of Pharmaceutical Sciences, Hokkaido University, Kita-12, Nishi-6, Kita-ku, Sapporo 060-0812,

Japan. E-mail: harasima@pharm.hokudai.ac.jp. Phone: +81-11-706-3919. Fax: +81-11-706-4879 


\section{Abstract}

The focus of this study was on the development of a nano carrier targeted to mitochondria, a promising therapeutic drug target. We synthesized a lipid derivative that is conjugated with a mitochondrial targeting signal peptide (MTS), which permits the selective delivery of certain types of proteins to mitochondria. We then explored the use of an innovative technology in which MTS and the MITO-Porter were integrated. The latter is a liposome that delivers cargos to mitochondria via membrane fusion. The results indicate that the combination of MTS and the MITO-Porter would be useful for selective mitochondrial delivery via membrane fusion.

Keywords: Mitochondria; Mitochondrial drug delivery; Mitochondrial targeting signal peptide (MTS);

MITO-Porter; Membrane fusion; Nanotechnology. 


\section{Introduction}

The efficient and site-specific delivery of therapeutic drugs is a critical issue in clinical nanotechnology. Receptor-targeted nanocarriers aimed at specific tissues and cells have been investigated by many researchers, and the findings have accelerated the application of nanotechnology in medicine (referred to as nanomedicine)(Juliano et al., 2009; Mohanty et al., 2011). However, these techniques have been limited to developing nanocarriers that target a specific organelle, such as the nucleus, mitochondria, golgi apparatus, endoplasmic reticulum, etc., which are promising targets for therapeutic drugs. Mitochondria play a role in the homeostasis of vital physiological functions, including electron transfer, apoptosis and calcium storage(Szewczyk and Wojtczak, 2002). Mitochondrial dysfunction is implicated in a variety of human diseases, including mitochondrial inherited diseases, neurodegenerative disorders, diabetes mellitus and cancer(Chan, 2006; Reeve et al., 2008; Schapira, 2006; Wallace, 2005), therefore, mitochondria would be expected to be the target organelle for these diseases (Armstrong, 2007; Du and Yan, 2010; Fulda et al., 2010; Giorgi et al., 2012; Gogvadze et al., 2009; Yamada et al., 2007).

Effective medical therapies for such diseases will ultimately require an optimal mitochondrial delivery system, in which multiple processes i.e., cellular uptake, endosomal escape and mitochondrial targeting should be regulated. Many researches regarding cellular uptake and endosomal escape to improve the intracellular trafficking of the carrier have been performed, because these topics are common issues in the development of drug delivery system(Miyata et al., 2012; Pack et al., 2005; Wang et al., 
2012; Yamada et al., 2012). On the other hand, the information about mitochondrial-targeted delivery of nano carrier has been limited, although many mitochondrial deliveries for various molecules have been reported(Mukhopadhyay and Weiner, 2007; Yamada and Harashima, 2008; Zhang et al., 2011), especially in the case of low molecular weight drugs(D'Souza et al., 2008; James et al., 2007; Wipf et al., 2005). The use of a mitochondrial targeting signal peptide (MTS) makes it possible to selectively deliver a variety of chemicals, proteins and linear DNA to mitochondria(Asayama et al., 2006; Flierl et al., 2003; Schatz, 1996). It is expected that MTS can be a useful ligand for selective mitochondrial delivery of nanoparticle. However, the direct modification of an MTS peptide to produce a nanoparticle for enhancing mitochondrial delivery has not been demonstrated.

The purpose of this study was to validate whether MTS can enhance the mitochondrial targeting of nanoparticles as well as endogenous mitochondrial proteins. We first synthesized a lipid derivative conjugated with MTS and then constructed MTS-modified liposomes. The mitochondrial binding activity of the prepared liposomes was then evaluated using isolated mitochondria. Mitochondrial targeting activity was also estimated using a cell homogenate, which is a better model of living cells than isolated mitochondria. Finally, we developed an innovative technology in which MTS and a MITO-Porter are integrated. The latter is a liposome-based nano carrier that delivers cargos to mitochondria via membrane fusion(Yamada et al., 2008; Yamada and Harashima, 2008; Yasuzaki et al., 2010) and verified the utility of the selectivity of MTS for mitochondrial delivery via membrane fusion. 



\section{Materials and methods}

\subsection{Materials}

Cholesterol (Chol), 1,2-Dioleoyl-sn-glycero-3-phosphatidyl ethanolamine (DOPE), 7-nitrobenz2-oxa-1, 3-diazole labeled DOPE (NBD-DOPE) and rhodamine-DOPE were purchased from Avanti Polar lipids (Alabaster, AL, USA). Egg yolk phosphatidyl choline (EPC) was obtained from Nippon Oil and Fats Co. (Tokyo, Japan). Sphingomyelin (SM) was purchased from Sigma (St. Louis, MO, USA). Stearyl octaarginine (STR-R8) was obtained from KURABO INDUSTRIES LTD (Osaka, Japan). MTS peptide (NH ${ }_{2}$ MVSGSSGLAAARLLSRTFLLQQNGIRHGSYC) was obtained from nippi incorporated (Tokyo, Japan) in the purified form. The pTENG and pTENMG plasmids encoding the green fluorescent protein (GFP) and MTS fused GFP used in the data shown in Figure 1 were constructed as described in Figure S1. Mitochondria were isolated from rat liver and homogenate solutions were prepared from HeLa cells essentially, as described in Supplementary material. All other chemicals used were commercially available reagent-grade products.

\subsection{Synthesis of MTS-DOPE}

The MTS peptide was conjugated to a DOPE lipid as shown in Figure 2A, using 4-(Nmaleimidomethyl)-cyclohexane-1-carboxylic acid N-hydroxysuccinimide ester (SMCC) as a bifunctional cross-linker. DOPE (1 eq.) and SMCC (1 eq.) were dissolved in $\mathrm{CHCl}_{3}$ in the presence of $\mathrm{Et}_{3} \mathrm{~N}$ (2 eq.) to synthesis the SMCC-DOPE conjugate. After stirring the reaction mixture at room temperature for $12 \mathrm{hr}$, 
a solution of MTS peptide in dimethyl sulfoxide (DMSO) was added to a mixture of SMCC-DOPE conjugate in $\mathrm{CHCl}_{3}(1: 2$ molar ratio) to produce the MTS-DOPE conjugate. The reaction mixture was stirred at room temperature for $1 \mathrm{hr}$ and the resulting solution was freeze-dried to give the crude MTSDOPE conjugate, followed by dissolution in $\mathrm{DMSO} / \mathrm{MeOH}(1: 3)$.

The crude MTS-DOPE conjugate was purified by reversed-phase high performance liquid chromatography (reversed-phase HPLC), using a COSMOSIL 5C18-AR-II column (4.6 x 250 mm, NACALAI TESQUE, INC, Kyoto, Japan) with a linear gradient of acetonitrile (30\% to $70 \%$ for 10 min, $90 \%$ to $100 \%$ for $10 \mathrm{~min}$ ) in an aqueous $0.1 \%$ trifluoroacetate solution (Fig. 2B). The HPLC profile was detected at $280 \mathrm{~nm}$. A new peak eluted at $21.5 \mathrm{~min}$, later than the unconjugated MTS (starting material, 8 min), and this peak was collected as an MTS-DOPE conjugate (Fig. 2B). The conjugation of MTS with DOPE was confirmed by determining the molecular weight of MTS-DOPE by matrix-assisted laser desorption ionization time of flight mass spectrometry (MALDI-TOF-MS) with Applied Biosystems Voyager System 1065 (Applera, Norwalk, CT) using acetonitrile : water $=1: 1$ with $0.3 \%$ trifluoroacetate as the matrix solution, supplied with $7 \mathrm{mg} / \mathrm{mL}$ of $\alpha$-Cyano-4-hydroxycinnamic acid.

\subsection{Preparation of Liposomes}

Liposomes composed of EPC/Chol (molar ratio: 9/2) and DOPE/SM (molar ratio: 9/2) were prepared by the lipid hydration method. A lipid film was formed by evaporation of the solvents (chloroform and ethanol) from a lipid solution in a glass tube. Then, $0.25 \mathrm{~mL}$ of mitochondrial isolation 
buffer [MIB: $250 \mathrm{mM}$ sucrose, $2 \mathrm{mM}$ Tris- $\mathrm{HCl}, \mathrm{pH}$ 7.4] was applied to $137.5 \mathrm{nmol}$ of dried lipid film.

After incubation for $10 \mathrm{~min}$ for hydration, the suspensions were sonicated using a bath-type sonicator ( 85

W, Aiwa Co., Tokyo, Japan) for $15 \mathrm{sec}$. To modify the prepared LPs with MTS or R8, the required amount of MTS-DOPE or STR-R8 was added to the lipid solution. Particle diameter was measured using a quasielastic light scattering method. The $\zeta$ potential was determined electrophoretically using laser doppler velocimetry (Zetasizer Nano ZS; Malvern Instruments, Herrenberg, Germany).

\subsection{Mitochondrial binding assay}

To determine the binding activity of the LP to mitochondria, the level of mitochondria-associated LP labeled with $1 \mathrm{~mol} \%$ NBD-DOPE was determined by fluorescence measurements. Labeled LP was added to mitochondrial suspension $(0.9 \mathrm{mg}$ of mitochondrial protein $/ \mathrm{mL}$ ) in $100 \mu \mathrm{L}$ of MIB (final lipid concentration of LP, $55 \mu \mathrm{M}$ ), and the suspension incubated for $30 \mathrm{~min}$ at $25^{\circ} \mathrm{C}$. The resulting solution was denoted as Sample A. The suspension was centrifuged at $16,000 \mathrm{x}$ g for $10 \mathrm{~min}$ at $4^{\circ} \mathrm{C}$ to precipitate the mitochondria, and the supernatant was removed. The pellets were washed twice with MIB and reprecipitated by centrifugation $\left(20,400 \mathrm{~g}, 4^{\circ} \mathrm{C}, 2 \mathrm{~min}\right)$. After removing the supernatant, the pellet was resuspended in $100 \mu \mathrm{L}$ of MIB to give Sample B. Samples A and B were solubilized with equal volumes of MIB containing $1 \%$ Triton $\mathrm{X}-100$, and the fluorescence intensities were measured with excitation at $470 \mathrm{~nm}$ and emission at $530 \mathrm{~nm}$. Binding activity (\%) was calculated as follows:

$$
\text { Binding activity }(\%)=\mathrm{F}_{\mathrm{B}} / \mathrm{F}_{\mathrm{A}} \times 100
$$

where $F_{A}$ and $F_{B}$ represent the fluorescence intensity of sample $A$ and sample $B$, respectively. 


\subsection{Evaluation of mitochondrial targeting activity in cell homogenate}

To evaluate mitochondrial targeting activity, LP labeled with 1 mol\% NBD-DOPE were added to homogenate solution, and then the level of fluorescence of the carriers in mitochondrial fraction and other organelle fractions were determined as described below. A $10-\mu \mathrm{L}$ aliquot of labeled liposome (lipid concentration, $550 \mu \mathrm{M}$ ) was added to homogenate in $90 \mu \mathrm{L}$ of MIB, and the resulting suspension incubated for $30 \mathrm{~min}$ at $25^{\circ} \mathrm{C}$. The resulting solution was denoted as Sample A. After incubation, each organelle fraction was obtained from the homogenate using a differential centrifugation technique. All subsequent steps were carried out on ice. The homogenate solution was centrifuged at $700 \mathrm{x} g$ for $10 \mathrm{~min}$ at $4^{\circ} \mathrm{C}$, and the pelleted fraction was resuspended in $100 \mu \mathrm{L}$ of MIB to give the $\mathrm{P}$ fraction, which contains nuclei and fracted cells. The supernatant was centrifuged at $16,000 \mathrm{~g}$ for $10 \mathrm{~min}$ at $4^{\circ} \mathrm{C}$, and the pelleted fraction was resuspended in $100 \mu \mathrm{L}$ of MIB to produce the mitochondrial fraction. The supernatant was centrifuged at 20,400g for $10 \mathrm{~min}$ at $4^{\circ} \mathrm{C}$, and the resulting supernatant was used as the $\mathrm{S}$ fraction contained light-density organelle and cytosol. Samples A and all fractions were solubilized with equal volumes of MIB containing 1\% Triton X-100, and the fluorescence intensities were measured with excitation at 470 $\mathrm{nm}$ and emission at $530 \mathrm{~nm}$. For the graph in Figure 4B, the percentage of carriers in mitochondrial, $\mathrm{P}$ and $\mathrm{S}$ fractions were estimated based on the fluorescence intensity of each fraction divided by that of total fraction. Mitochondrial targeting activity (\%) was calculated using the expression below:

Mitochondrial targeting activity $(\%)=\mathrm{F}_{\mathrm{mt}} / \mathrm{F}_{\mathrm{A}} \mathrm{x} 100$ 
where $\mathrm{F}_{\mathrm{A}}$ and $\mathrm{F}_{\mathrm{mt}}$ represent the fluorescence intensity of sample $\mathrm{A}$ and mitochondrial fraction, respectively.

\subsection{Membrane fusion assay using FRET analysis}

The membrane fusion activity of the liposomes with mitochondria was assessed by fluorescence resonance energy transfer (FRET), as described previously(Maier et al., 2002; Struck et al., 1981; Yamada et al., 2008). In this experiment, liposomes labeled with both 1 mol\% NBD-DOPE and $0.5 \mathrm{~mol} \%$ rhodamine-DOPE were used. A 10- $\mu \mathrm{L}$ aliquot of labeled liposome (lipid concentration, $550 \mu \mathrm{M}$ ) was added to mitochondrial suspension $(0.9 \mathrm{mg}$ of mitochondrial protein $/ \mathrm{mL})$ in $90 \mu \mathrm{L}$ of MIB, and incubated for $30 \mathrm{~min}$ at $25^{\circ} \mathrm{C}$. After incubation, the fluorescent spectra were obtained by measuring the fluorescent intensity in the range from 500 to $650 \mathrm{~nm}$ (excitation at $470 \mathrm{~nm}$ ). Membrane fusion between the labeled liposomes and the mitochondria will lead to the diffusion of NBD and rhodamine into the lipid membranes, which causes a reduction in energy transfer, resulting in an increase in fluorescence intensity at $530 \mathrm{~nm}$. The maximum fluorescence was defined as the fluorescence of liposomes when dissolved in Triton X100 (final concentration, $0.5 \%(\mathrm{v} / \mathrm{v})$ ). The experimental protocol is summarized in Figure S4A. Fusion activity (\%) was estimated by the reduction in the level of energy transfer in accordance with membrane fusion, and was calculated as follows:

Fusion activity $(\%)=\left(F-F_{0}\right) /\left(F_{\max }-F_{0}\right) \times 100$

where $F, F_{0}$ and $F_{\max }$ represent the fluorescence intensity of labeled liposome after incubation with mitochondria, the fluorescence intensity of labeled liposome after incubation without mitochondria, and the maximum fluorescence intensity after the Triton X-100 treatment, respectively. 


\section{Results}

\subsection{Synthesis of MTS-DOPE}

In this experiment, we used the MTS peptide derived from rat succinyl CoA synthetase (Henning et al., 1988) as a mitochondrial targeting ligand. We first confirmed that this MTS is functional in human cells, because we planned to investigate mitochondrial targeting activity using a cell homogenate prepared with human HeLa cells. The pTENG and pTENMG plasmids encoding the GFP and the MTS fused GFP were transfected into HeLa cells as described in the Supplementary Material. After transfection, the HeLa cells were observed by confocal laser scanning microscopy (Fig. 1). The intracellular observation showed that the MTS-GFP became localized in mitochondria (Fig. 1A). On the other hand, GFP had diffused throughout the cells (Fig. 1B). Based on these results, we concluded that the MTS derived from rat can selectively deliver exogenous protein deliver to mitochondria, even in human HeLa cells.

To display MTS on the surface of a liposome, a lipid derivative conjugated with MTS (MTSDOPE) was synthesized as shown in Figure 2A. After the reaction for synthesis of the MTS-DOPE, the product was purified by reverse-phased HPLC. The HPLC-chart permitted us to confirm successful conjugation of the MTS peptide with the DOPE lipid (Fig. 2B, Fig. S2A). A new peak eluting at $21.5 \mathrm{~min}$, slower than the unconjugated MTS peptide (starting material, 8 min), was collected as an MTS-DOPE. The slower eluting compound corresponded to the conjugation of the hydrophobic DOPE lipid. We also observed the unconjugated SMCC-DOPE at around $25 \min$ (data not shown), however we couldn't detect unreacted DOPE and SMCC. To further confirm that the MTS peptide was conjugated to the DOPE lipid, 
MALDI-TOF MS analyses were performed for the MTS peptide (calculated MW3292.7 and observed MW3292.7 in Fig. S2B) and MTS-DOPE (calculated MW4254.3 and observed MW4255.0 in Fig. 2C). These mass analysis data indicate that the conjugation was successful, as evidenced by the molecular shifts in the MALDI-TOF MS analysis.

\subsection{Construction of MTS modified liposomes and their mitochondrial binding activities using isolated} mitochondria

We constructed the MTS-modified liposomes containing EPC/Chol (9:2) (MTS-EPC-LP) using synthesized MTS-DOPE and hydration method. The modified liposomes were prepared by incorporating MTS-DOPE at levels of 1, 2.5, 5, $10 \mathrm{~mol} \%$ of the total lipid. The prepared liposomes were comparable in their diameters (approximately 200-300 nm, Table 1). On the other hand, the addition of the MTS peptide resulted in a gradual increase in the surface charge of the liposomes, and the surface-charge became saturated when the concentration of MTS-DOPE exceeded 5mol\% of total lipids (Fig. 3A). We next performed a mitochondrial binding assay of MTS-EPC-LPs using isolated mitochondria. The results indicated that the MTS peptide enhanced the mitochondrial binding of liposomes with isolated mitochondria dependent on increase in MTS-concentration (Fig. 3B). These results suggest that MTS is displayed on the surface of liposomes and it functions as a mitochondrial targeting ligand. 


\subsection{Evaluation of the mitochondrial targeting activity of MTS-EPC-LP using a homogenate}

We evaluated the mitochondrial targeting activity of the MTS-modified liposomes to ascertain whether MTS facilitates the interaction between the liposomes and mitochondria under conditions where other organelles are present, along with mitochondria. In this experiment, we also investigated the mitochondrial targeting activity of liposomes that had been modified with R8, which greatly assist in binding between isolated mitochondria and the carrier(Yamada and Harashima, 2008). The experimental scheme is shown in Figure 4A. Liposomes labeled with a fluorescent dye (Probe-LPs) were added to the cell homogenate, and the fluorescent level of the carriers in the mitochondrial fraction and other organelle fractions was then measured after subcellular fractionation. The P fraction contained nuclei and fracted cells, and the $\mathrm{S}$ fraction contained light-density organelle and cytosol. The percentages of liposomes in the mitochondrial, $\mathrm{P}$ and $\mathrm{S}$ fractions are summarized in Figure 4B, when liposomes (EPC/Chol =9:2) modified with 5 mol\% MTS (MTS-EPC-LP), 5 mol\% R8 (R8-EPC-LP) or non-modified liposomes (EPCLP) were used.

In the case of MTS-EPC-LP, the percentage of mitochondria-bound liposomes to the total amount applied was approximately 30\% (Fig. 4B). We also observed that numerous MTS-EPC-LPs were present in the $\mathrm{P}$ fraction, where mitochondria were present in a fracted cell with a disrupted plasma membrane. We presumed that MTS-EPC-LP would not bind to pure mitochondria, when TOM/TIM complexs (MTS binding sites) on the outer membranes had been capped by the carriers. Accordingly, the rest of the carriers might have access to mitochondria in the case of fracted cells. In the case of R8-EPC- 
LP, the mitochondrial targeting activity was comparable with that of non-modified liposomes in homogenate solution, although the mitochondrial binding activity was very high in the evaluation experiments using an isolated mitochondria suspension (Table S1, Fig. S3). The results suggest that the cationic R8 peptide might bind to mitochondria and other organelles via non-specific electrostatic interactions.

3.4. Construction of MTS modified MITO-Porter and its mitochondrial targeting activities in a homogenate

The findings of the present study indicated that MTS enhanced the mitochondrial targeting of nanoparticles that are much larger than even liposomes with a diameter of $200 \mathrm{~nm}$ (Fig. 4B). However, the major remaining problem in mitochondrial delivery is whether the cargo could be delivered across mitochondrial membranes. Mitochondrial delivery using MTS fails in cases where macromolecules and hydrophobic molecules are transported(Endo et al., 1995; Esaki et al., 1999; Gruhler et al., 1995), because the MTS must pass through the narrow space composed of the TOM/TIM complex in mitochondrial membranes. Here, we verified the utility of the combination between MTS for selective mitochondrial targeting and mitochondrial macromolecule delivery using a MITO-Porter(Yamada et al., 2008; Yamada and Harashima, 2008; Yasuzaki et al., 2010). Using the GFP as a model macromolecule and analysis by confocal laser scanning microscopy, we were able to confirm the delivery of macromolecules to mitochondria using the MITO-Porter ${ }^{9,18-19}$. 
We prepared a MTS-modified MITO-Porter (DOPE/SM =9:2), and compared the mitochondrial targeting activity with R8-modifeid MITO-Porter (DOPE/SM =9:2), a conventional MITO-Porter described in previous our reports(Yamada et al., 2008; Yamada and Harashima, 2008; Yasuzaki et al., 2010). The prepared liposomes were comparable in their diameters (approximately 150-200 nm) as shown in Table 2. A mitochondrial targeting assay using a cell homogenate showed that MTS-modified MITOPorter targeted mitochondria more efficiently than the R8-modified MITO-Porter (Fig. 5). As shown in Figure 4B, it was also observed that MTS enhanced mitochondrial targeting in cell homogenates, when the liposomes $(\mathrm{EPC} / \mathrm{Chol}=9: 2)$ with low mitochondrial fusion activity were used. These results indicate that mitochondrial targeting was not affected by the lipid composition of the liposomal carriers.

\subsection{Evaluation for mitochondrial membrane fusion activity of MTS modified MITO-Porter}

We investigated the mitochondrial membrane fusion activity of the MTS-modified MITO-Porter.

Figure 6A shows the schematic image regarding the membrane fusion analysis using FRET. Liposomal membranes were labeled with both NBD-DOPE (excitation at $460 \mathrm{~nm}$ and emission at $534 \mathrm{~nm}$ ) and rhodamine-DOPE (excitation at $550 \mathrm{~nm}$ and emission at $590 \mathrm{~nm}$ ) so that energy transfer will occur from NBD to rhodamine. Membrane fusion between the labeled liposomes and the mitochondria will lead to the diffusion of NBD and rhodamine into the lipid membranes, which causes a reduction in energy transfer, resulting in an increase in fluorescence intensity at $530 \mathrm{~nm}$. 
We observed that the fluorescence intensity at $530 \mathrm{~nm}$ increased in the case of the liposomes modified with MTS or R8 (Fig. S4B a), S4B b)), indicating a reduction in energy transfer due to fusion between the liposomal and mitochondrial membranes. Whereas no such increase at $530 \mathrm{~nm}$ was detected in the case of non-modified liposomes (Fig. S4B c)). The fusion activity with mitochondrial membranes was calculated, based on the spectra and the results indicate that the MTS-modified MITO-Porters and R8-modified MITO-Porters had a significantly higher fusogenic activity than non-modified liposomes (Fig. 6B). The findings reported herein suggest that a combination between MTS and MITO-Porter would be useful for selective mitochondrial delivery of macromolecules via membrane fusion. 


\section{Discussion}

In the previous study, we prepared R8-modified liposomes by just mixing of liposomes and stearyl

R8 solutions(Yamada et al., 2008). We first attempted to prepare MTS-modified liposomes using the same procedure, however, the methods were not optimal in this case, because the MTS-DOPE was not soluble in water but in an organic solvent. Therefore, we prepared MTS-modified liposomes using lipid films containing MTS-DOPE formed by evaporation of the organic solvents in a glass tube, by the hydration method to produce multi-lamellar vesicles. The MTS peptides would probably be present in both sides of the liposomal membranes in the prepared multi-lamellar vesicles.

The preparation method permitted the construction of MTS-modified liposomes including EPC or DOPE, with diameters of approximately 200-300 nm (Table 1, Table 2). However, aggregation occurred frequently, when the amount of MTS-DOPE was increased, and it was not possible to prepare $10 \mathrm{~mol} \%$ of MTS-modified liposomes containing the DOPE, which forms a highly fluid lipid membrane. Presumably, the MTS peptide might easily interact with each other on liposomal membranes, because the peptide is a hydrophobic peptide with cationic charges having detergent-like properties. Thus, it may be difficult for the MTS-modified liposomes to maintain their structures in the presence of high densities of the MTS peptide.

In this study, we focused on mitochondrial targeting of nanoparticles, and evaluated the mitochondrial selectivity of MTS when the liposomal surface was modified with the peptide. Mitochondrial targeting analysis showed that MTS can function as a mitochondrial targeting device for 
liposomes (Fig. 3B, Fig. 4B, Fig. 5), although the physical properties of liposomes are quite different from that of proteins, which are original cargoes of MTS. On the other hand, we observed that the almost half of the carriers remained in other compartments (Fig. 4B). To verify that MTS on the nanoparticle-surface functions adequately with the TOM/TIM complex on the outer membranes of mitochondria, an innovative design for the construction of MTS-modified nanoparticles is needed. We are planning to improve the mitochondrial selectivity of MTS-modified nanoparticles, by focusing on the flexibility of MTS on the nanoparticle-surface.

To achieve mitochondrial-targeted delivery by nanoparticles, multiple processes including cellular uptake, endosomal escape and mitochondrial targeting should be required. In this study, we showed that MTS enhanced the mitochondrial targeting of nanoparticles in cell homogenate (Fig. 4B, Fig. 5), and also indicated that the MTS-modified MITO-Porter could efficiently fuse with isolated mitochondria (Fig. 6B). In previous studies, we developed a Dual Function (DF)-MITO-Porter, which possesses mitochondriafusogenic inner envelopes and endosome-fusogenic outer envelopes (Yamada et al., 2011; Yamada and Harashima, 2012). Intracellular observations showed that the DF-MITO-Porter effectively delivered exogenous bioactive macromolecules into the mitochondria in living cells (Yamada et al., 2011; Yamada and Harashima, 2012). Future study will include the packaging of MTS-modified MITO-Porter via endosome-fusogenic envelopes to achieve mitochondrial selective targeting in living cells using DFMTS-modified MITO-Porter. 


\section{Conclusion}

Organelle targeting is one of the challenges in drug delivery. In this study, we reported an approach

for mitochondrial targeting using surface modification of liposomes with an MTS peptide. Efficient accumulation of the MTS-modified liposomes on mitochondria was demonstrated using cell homogenate cocktails. In combination with mitochondrial fusion liposomes, MITO-Porter, the highly mitochondrial membrane fusion activity was indicated. These results would support our approach that a combination between MTS for selective mitochondrial targeting and mitochondrial delivery via membrane fusion has considerable potential for such targeting. In the future, we will plan to improve the MTS-modified MITOPorter, focusing on the environment of MTS on nanoparticle-surface and the cytoplasmic delivery. Studies directed at this goal are currently underway. 


\section{Acknowledgment}

This work was supported, in part by, the Program for Promotion of Fundamental Studies in Health

Sciences of the National Institute of Biomedical Innovation, Japan (NIBIO), a Grant-in-Aid for Young

Scientists (A) and a Grant-in-Aid for Scientific Research (S) from the Ministry of Education, Culture,

Sports, Science and Technology of Japanese Government (MEXT). We also thank Milton Feather for his

helpful advice in writing the manuscript. 


\section{References.}

Armstrong, J.S., 2007. Mitochondrial medicine: Pharmacological targeting of mitochondria in disease. Brit J Pharmacol 151, 1154-1165.

Asayama, S., Kawamura, E., Nagaoka, S., Kawakami, H., 2006. Design of manganese porphyrin modified with mitochondrial signal peptide for a new antioxidant. Mol Pharm 3, 468-470.

Chan, D.C., 2006. Mitochondria: dynamic organelles in disease, aging, and development. Cell 125, 1241 1252.

D'Souza, G.G.M., Cheng, S.M., Boddapati, S.V., Horobin, R.W., Weissig, V., 2008. Nanocarrier-assisted sub-cellular targeting to the site of mitochondria improves the pro-apoptotic activity of paclitaxel. J Drug Target 16, 578-585.

Du, H., Yan, S.S.D., 2010. Mitochondrial medicine for neurodegenerative diseases. Int J Biochem Cell B 42, 560-572.

Endo, T., Nakayama, Y., Nakai, M., 1995. Avidin fusion protein as a tool to generate a stable translocation intermediate spanning the mitochondrial membranes. J Biochem (Tokyo) 118, 753-759.

Esaki, M., Kanamori, T., Nishikawa, S., Endo, T., 1999. Two distinct mechanisms drive protein translocation across the mitochondrial outer membrane in the late step of the cytochrome $b(2)$ import pathway. Proc Natl Acad Sci U S A 96, 11770-11775.

Flierl, A., Jackson, C., Cottrell, B., Murdock, D., Seibel, P., Wallace, D.C., 2003. Targeted delivery of DNA to the mitochondrial compartment via import sequence-conjugated peptide nucleic acid. Mol Ther 7, 550-557.

Fulda, S., Galluzzi, L., Kroemer, G., 2010. Targeting mitochondria for cancer therapy. Nat Rev Drug Discov 9, 447-464.

Giorgi, C., Agnoletto, C., Bononi, A., Bonora, M., De Marchi, E., Marchi, S., Missiroli, S., Patergnani, S., Poletti, F., Rimessi, A., Suski, J.M., Wieckowski, M.R., Pinton, P., 2012. Mitochondrial calcium homeostasis as potential target for mitochondrial medicine. Mitochondrion 12, 77-85.

Gogvadze, V., Orrenius, S., Zhivotovsky, B., 2009. Mitochondria as targets for cancer chemotherapy. Semin Cancer Biol 19, 57-66.

Gruhler, A., Ono, H., Guiard, B., Neupert, W., Stuart, R.A., 1995. A novel intermediate on the import pathway of cytochrome b2 into mitochondria: evidence for conservative sorting. Embo J 14, 1349-1359. Henning, W.D., Upton, C., McFadden, G., Majumdar, R., Bridger, W.A., 1988. Cloning and sequencing of the cytoplasmic precursor to the alpha subunit of rat liver mitochondrial succinyl-CoA synthetase. Proc Natl Acad Sci U S A 85, 1432-1436.

James, A.M., Sharpley, M.S., Manas, A.R., Frerman, F.E., Hirst, J., Smith, R.A., Murphy, M.P., 2007. Interaction of the mitochondria-targeted antioxidant MitoQ with phospholipid bilayers and ubiquinone oxidoreductases. J Biol Chem 282, 14708-14718.

Juliano, R.L., Alam, R., Dixit, V., Kang, H.M., 2009. Cell-targeting and cell-penetrating peptides for delivery of therapeutic and imaging agents. Wiley Interdiscip Rev Nanomed Nanobiotechnol 1, 324-335. Maier, O., Oberle, V., Hoekstra, D., 2002. Fluorescent lipid probes: some properties and applications (a review). Chem Phys Lipids 116, 3-18. 
Miyata, K., Nishiyama, N., Kataoka, K., 2012. Rational design of smart supramolecular assemblies for gene delivery: chemical challenges in the creation of artificial viruses. Chem Soc Rev 41, 2562-2574.

Mohanty, C., Das, M., Kanwar, J.R., Sahoo, S.K., 2011. Receptor mediated tumor targeting: an emerging approach for cancer therapy. Curr Drug Deliv 8, 45-58.

Mukhopadhyay, A., Weiner, H., 2007. Delivery of drugs and macromolecules to mitochondria. Adv Drug Deliv Rev 59, 729-738.

Pack, D.W., Hoffman, A.S., Pun, S., Stayton, P.S., 2005. Design and development of polymers for gene delivery. Nat Rev Drug Discov 4, 581-593.

Reeve, A.K., Krishnan, K.J., Turnbull, D., 2008. Mitochondrial DNA mutations in disease, aging, and neurodegeneration. Ann N Y Acad Sci 1147, 21-29.

Schapira, A.H., 2006. Mitochondrial disease. Lancet 368, 70-82.

Schatz, G., 1996. The protein import system of mitochondria. J Biol Chem 271, 31763-31766.

Struck, D.K., Hoekstra, D., Pagano, R.E., 1981. Use of resonance energy transfer to monitor membrane fusion. Biochemistry 20, 4093-4099.

Szewczyk, A., Wojtczak, L., 2002. Mitochondria as a pharmacological target. Pharmacol Rev 54, 101 127.

Wallace, D.C., 2005. The mitochondrial genome in human adaptive radiation and disease: on the road to therapeutics and performance enhancement. Gene 354, 169-180.

Wang, T., Upponi, J.R., Torchilin, V.P., 2012. Design of multifunctional non-viral gene vectors to overcome physiological barriers: Dilemmas and strategies. Int J Pharmaceut 427, 3-20.

Wipf, P., Xiao, J., Jiang, J., Belikova, N.A., Tyurin, V.A., Fink, M.P., Kagan, V.E., 2005. Mitochondrial targeting of selective electron scavengers: synthesis and biological analysis of hemigramicidin-TEMPO conjugates. J Am Chem Soc 127, 12460-12461.

Yamada, Y., Akita, H., Harashima, H., 2012. Multifunctional envelope-type nano device (MEND) for organelle targeting via a stepwise membrane fusion process. Methods Enzymol 509, 301-326.

Yamada, Y., Akita, H., Kamiya, H., Kogure, K., Yamamoto, T., Shinohara, Y., Yamashita, K., Kobayashi, H., Kikuchi, H., Harashima, H., 2008. MITO-Porter: A liposome-based carrier system for delivery of macromolecules into mitochondria via membrane fusion. Biochimica et biophysica acta 1778, 423-432.

Yamada, Y., Akita, H., Kogure, K., Kamiya, H., Harashima, H., 2007. Mitochondrial drug delivery and mitochondrial disease therapy - An approach to liposome-based delivery targeted to mitochondria. Mitochondrion 7, 63-71.

Yamada, Y., Furukawa, R., Yasuzaki, Y., Harashima, H., 2011. Dual function MITO-Porter, a nano carrier integrating both efficient cytoplasmic delivery and mitochondrial macromolecule delivery. Molecular therapy : the journal of the American Society of Gene Therapy 19, 1449-1456.

Yamada, Y., Harashima, H., 2008. Mitochondrial drug delivery systems for macromolecule and their therapeutic application to mitochondrial diseases. Adv Drug Deliv Rev 60, 1439-1462.

Yamada, Y., Harashima, H., 2012. Delivery of bioactive molecules to the mitochondrial genome using a membrane-fusing, liposome-based carrier, DF-MITO-Porter. Biomaterials 33, 1589-1595. 
Yasuzaki, Y., Yamada, Y., Harashima, H., 2010. Mitochondrial matrix delivery using MITO-Porter, a liposome-based carrier that specifies fusion with mitochondrial membranes. Biochem Biophys Res Commun 397, 181-186.

Zhang, E., Zhang, C., Su, Y., Cheng, T., Shi, C., 2011. Newly developed strategies for multifunctional mitochondria-targeted agents in cancer therapy. Drug Discov Today 16, 140-146. 


\section{Figure legends}

Fig. 1. Intracellular observation of MTS-GFP and GFP in human HeLa cells. MTS fused GFP (MTSGFP) and GFP and were expressed in HeLa cells by transfection of the pTENMG and pTENG plasmids, which encode the MTS-GFP and GFP. The MTS was derived from rat succinyl-CoA synthetase. The intracellular trafficking of MTS-GFP (A) and GFP (B) were observed using confocal laser scanning microscopy.

Fig. 2. Synthesis of MTS-DOPE and the characteristics. (A) Scheme for the synthesis of MTS-DOPE. (B) Behavior upon reverse-phased HPLC for the reaction mixture of MTS-DOPE detected at $280 \mathrm{~nm}$. HPLC conditions, mobile phase flowing at $1 \mathrm{~mL} / \mathrm{min}$ : solvent $\mathrm{A}(0.1 \%$ trifluoroacetate aqueous solution $)$, solvent B (0.1\% trifluoroacetate acetonitrile solution), 0-10 min linear gradient from $30-70 \% \mathrm{~B}, 10-20$ min linear gradient from $90-100 \% \mathrm{~B}$ and later isocratic condition of $100 \%$ B. (C) Molecular weight analysis of MTSDOPE by MALDI-TOF-MS. The calculated molecular weight of conjugated MTS-DOPE is 4254.3.

Fig. 3. Characteristics of MTS-modified liposomes. (A) The $\zeta$ potential of liposomes (EPC/Chol=9:2) conjugated with MTS-peptide at various molar ratios. (B) Mitochondrial binding activity of liposomes (EPC/Chol=9:2) conjugated with MTS-peptide at various molar ratio. Data are means \pm S.D. $(n=3-6)$. Significant differences between non-modified liposomes and MTS-modified liposomes $(\mathrm{p}<0.01$ by oneway ANOVA, followed by Bonferroni correction). 
Fig. 4. Evaluation of mitochondrial targeting activity of MTS modified EPC-LPs in a cell homogenate.

(A) Schematic image of the mitochondrial targeting assay. Liposomes labeled with a fluorescent dye (Probe-LPs) were added to a cell homogenate and the fluorescent level of the carriers in the mitochondrial fraction and other organelle fractions were measured after subcellular fractionation. The $\mathrm{P}$ fraction contained nuclei and fracted cells, and the S fraction contained low-density organelles and cytosol. (B) The percentage of liposomes in the mitochondrial, $\mathrm{P}$ and $\mathrm{S}$ fractions, when liposomes (EPC/Chol=9:2) modified with MTS (MTS-EPC-LP), R8 (R8-EPC-LP), or non-modified liposome (EPC-LP) were used.

Fig. 5. Evaluation of mitochondrial targeting of MTS modified MITO-Porter in a cell homogenate. Mitochondrial targeting activity of liposomes (DOPE/SM=9:2) conjugated with MTS-peptide (MTSMITO-Porter), R8-peptide (R8-MITO-Porter) and non-modified liposomes (DOPE-LP). Data are the means \pm S.D. $(n=3-4)$. Significant difference between DOPE-LP and others $(p<0.01$ by one-way ANOVA, followed by Bonferroni correction).

Fig. 6. Evaluation of mitochondrial membrane fusion of MTS modified MITO-Porter. (A) Schematic image of the membrane fusion analysis using FRET. Liposomal membranes were labeled with both NBD and rhodamine so that energy transfer will occur from NBD to rhodamine. Membrane fusion between the 
labeled liposomes and the mitochondria will lead to the diffusion of NBD and rhodamine into the lipid membranes, which causes a reduction in energy transfer. (B) Mitochondrial membrane fusion activity of liposomes (DOPE/SM=9:2) conjugated with MTS-peptide (MTS-MITO-Porter), R8-peptide (R8-MITOPorter) and non-modified liposomes (DOPE-LP). Data are means \pm S.D. $(n=3-4)$. Significant difference between DOPE-LP and others $(* \mathrm{p}<0.05, * * \mathrm{p}<0.01$ by one-way ANOVA, followed by Bonferroni correction).

Table 1. Diameters of MTS modified liposomes composed of EPC and Chol

Data are means \pm S.D. $(n=3-6)$

Table 2. Diameters of MTS-modified MITO-Porter

Data are means \pm S.D. $(n=3-4)$ 
Fig. 1

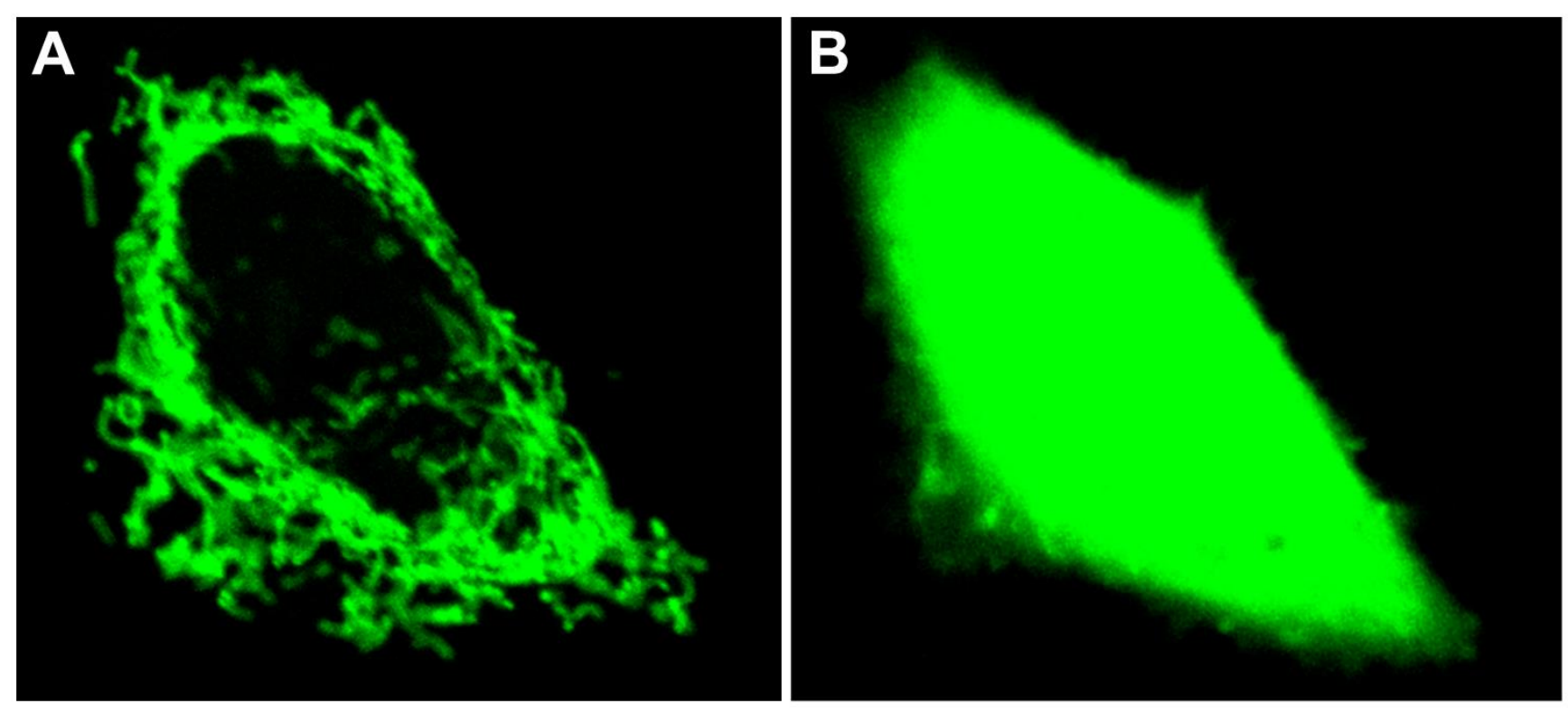


Fig. 2
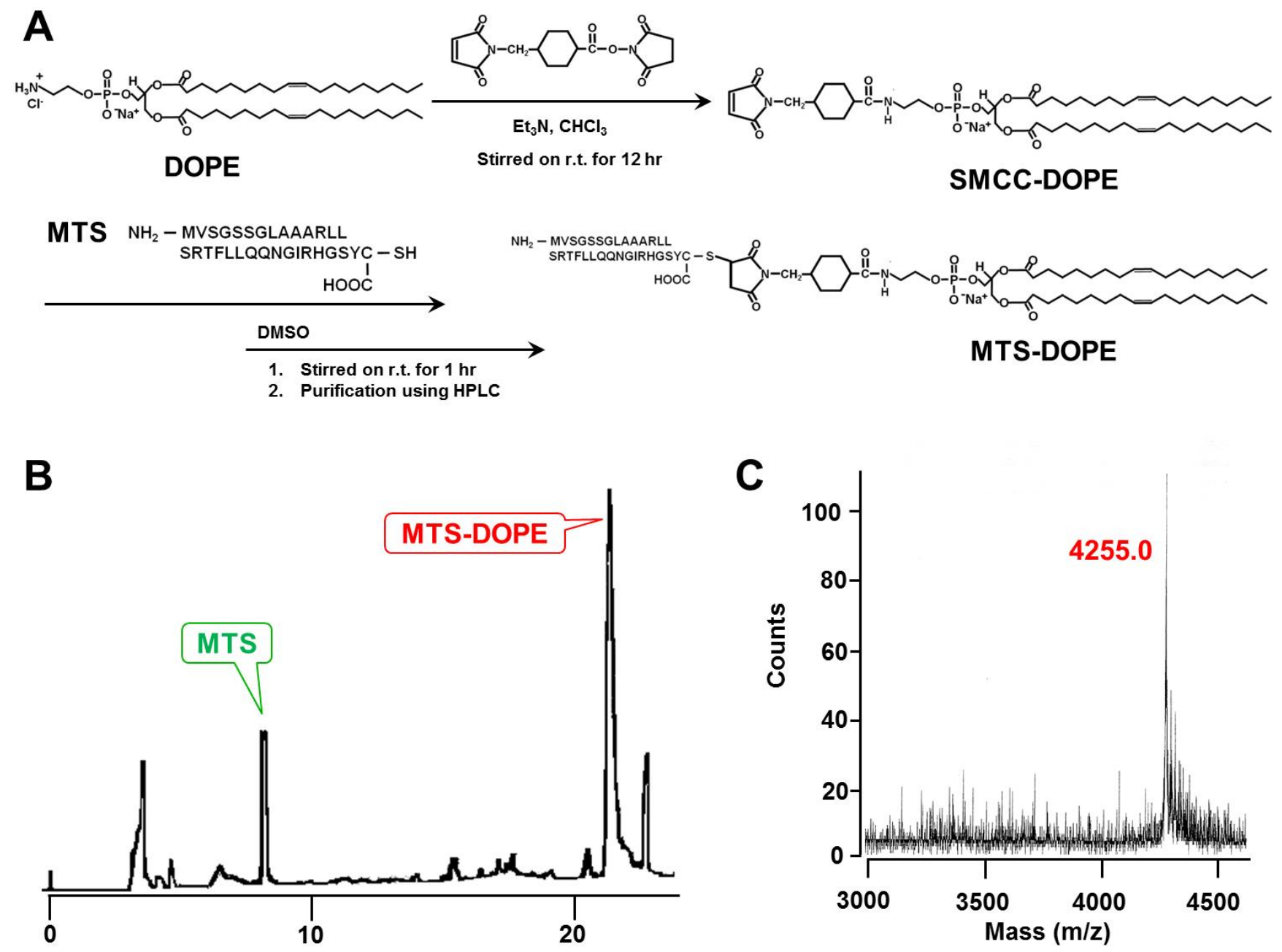
Fig. 3
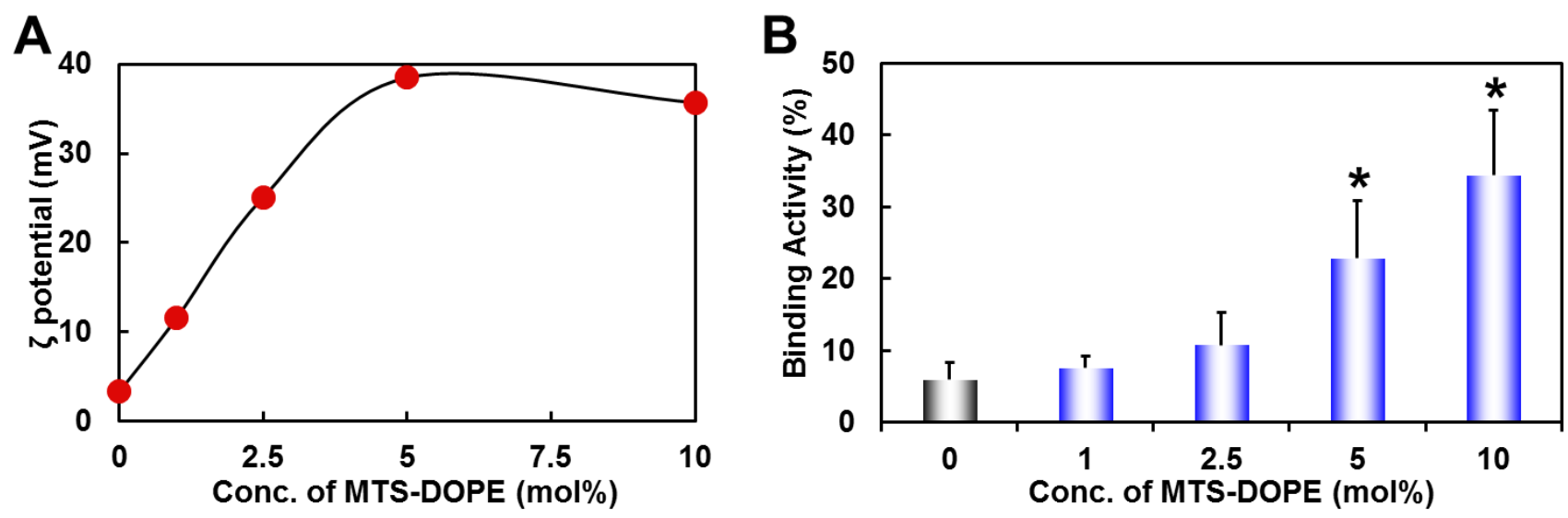
Fig. 4

A

1. Addition of LP to 2. Incubation organelle cocktail $\left(30 \mathrm{~min}, 2^{\circ} \mathrm{C}\right)$

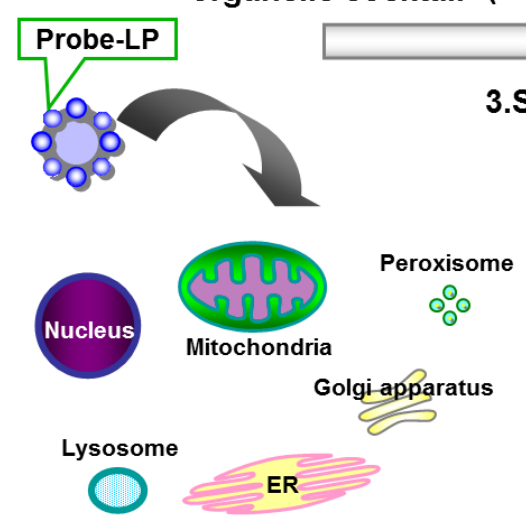

B

a) MTS-EPC-LP

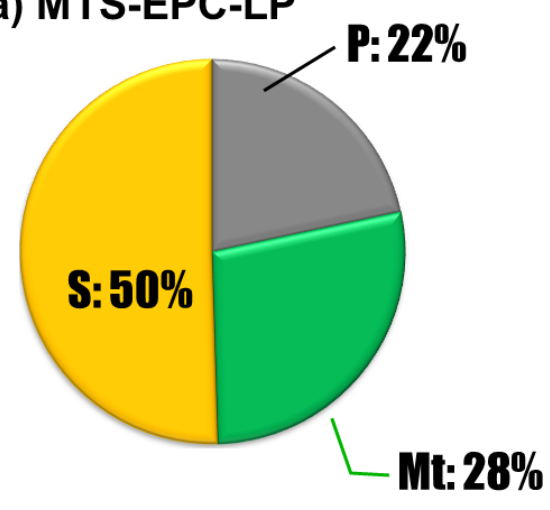

b) R8-EPC-LP

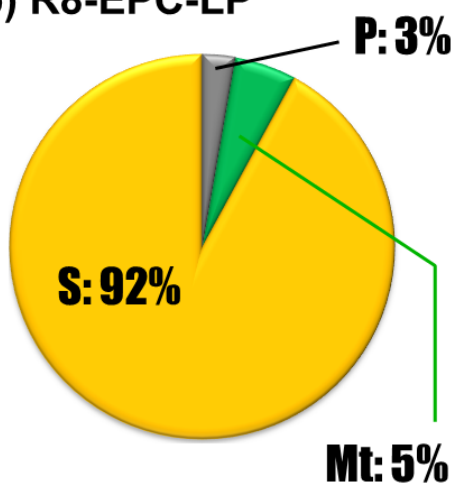

4. Measurement of

Fluorescence Intensity (FI)

5. Calculation of

Targeting Activity (\%)

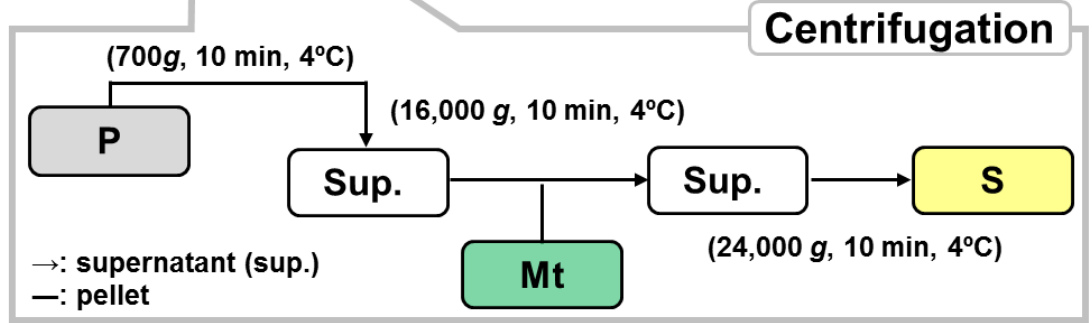

c) ECP-LP

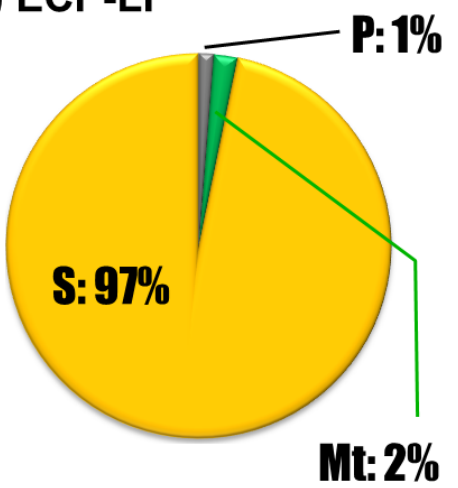


Fig. 5

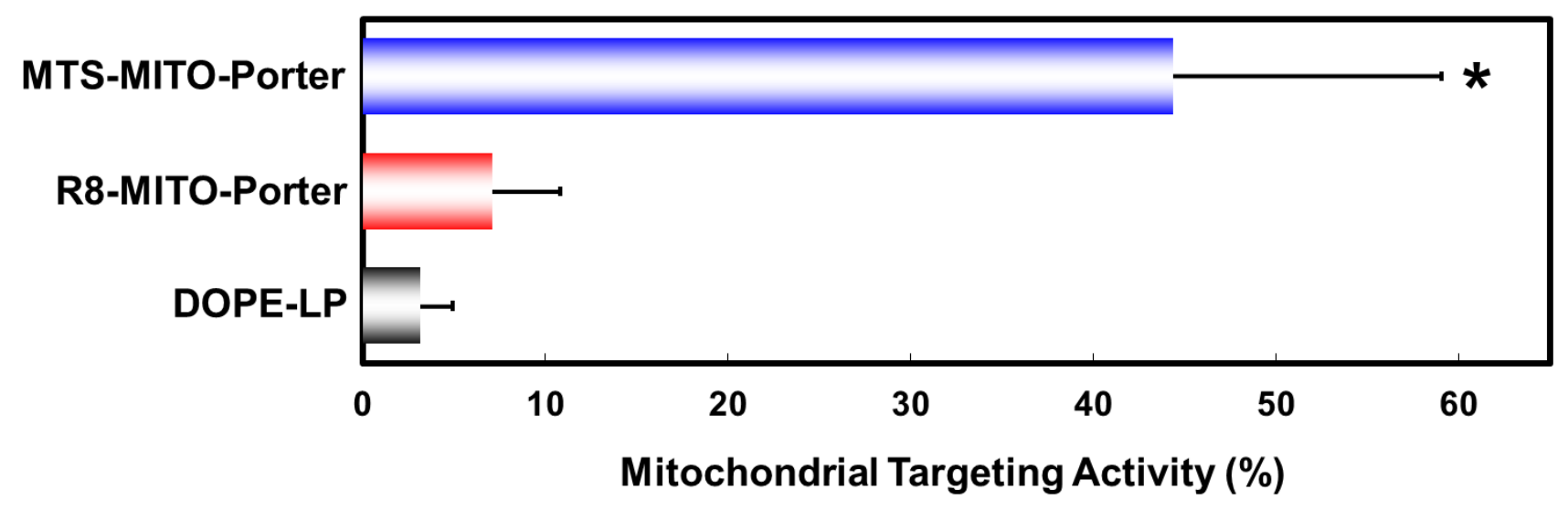


Fig. 6
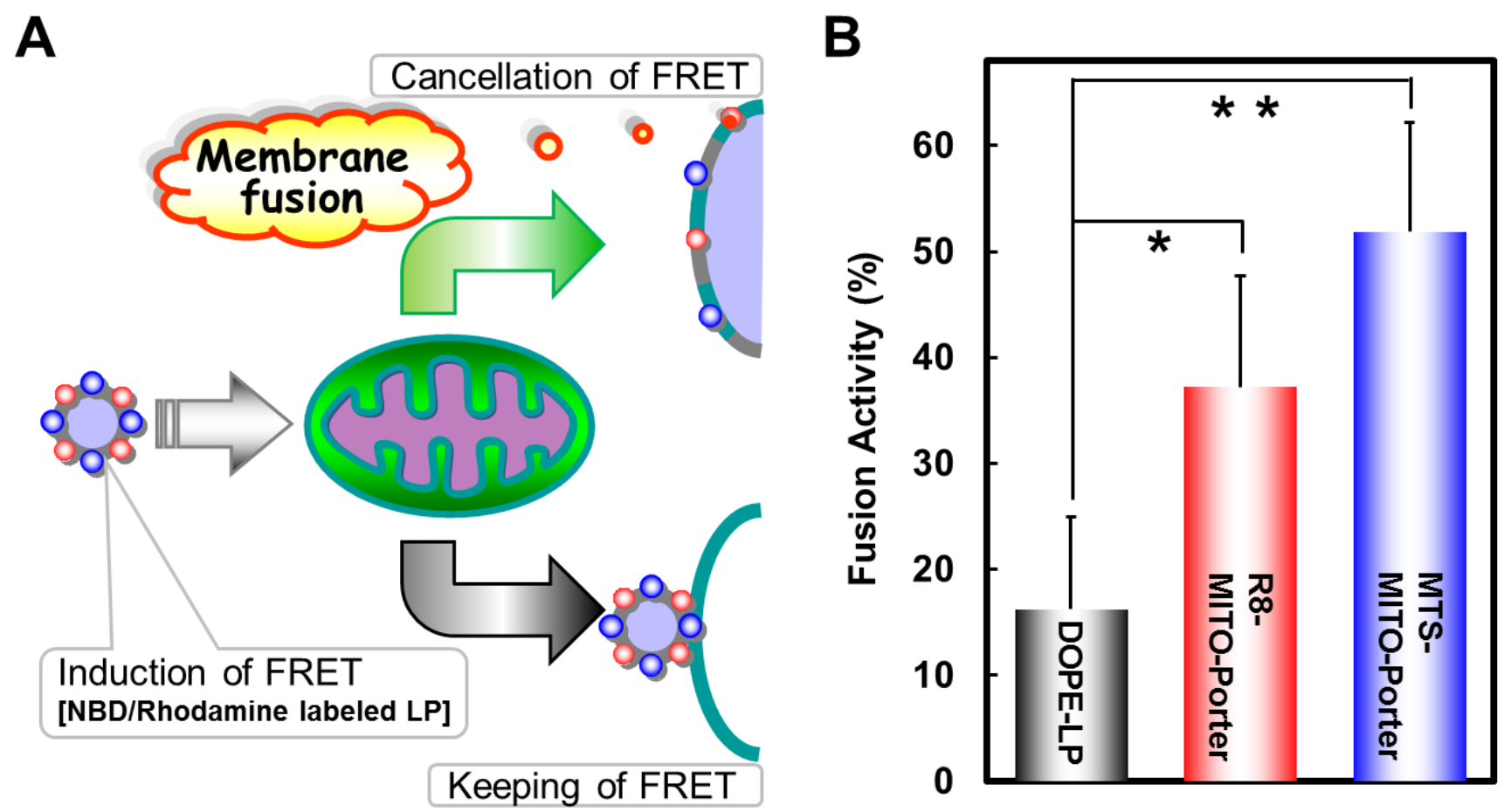


\section{Table 1}

Table 1. Diameters of MTS modified liposomes composed of EPC and Chol

\begin{tabular}{|c|c|c|c|c|c|}
\hline & \multicolumn{5}{|c|}{ Conc. of MTS-DOPE (mol\%) } \\
\cline { 2 - 6 } & 0 & 1 & 2.5 & 5 & 10 \\
\hline EPC/Chol (9:2) & $183 \pm 49$ & $197 \pm 31$ & $293 \pm 29$ & $237 \pm 89$ & $219 \pm 74$ \\
\hline
\end{tabular}

Data are means \pm S.D. $(n=3-6)$ 
Table 2

\section{Table 2. Diameters of MTS-modified MITO-Porter}

\begin{tabular}{|c|c|c|}
\hline $\begin{array}{c}\text { MTS-modified } \\
\text { MITO-Porter }\end{array}$ & $\begin{array}{c}\text { R8-modified } \\
\text { MITO-Porter }\end{array}$ & DOPE-LP \\
\hline \hline $204 \pm 22$ & $168 \pm 23$ & $215 \pm 37$ \\
\hline
\end{tabular}

Data are means \pm S.D. $(n=3-4)$ 


\section{Supporting Information}

\section{Materials and Methods}

\section{Materials}

Adult male Wistar rats (6-10 weeks old) were purchased from Sankyo Labo Service (Sapporo, Japan). GFP expression vector (pTENG) was prepared as described in our precious report(Mohanty et al., 2011). pDNA was purified using a Qiagen EndoFree Plasmid Mega Kit (Qiagen GmbH, Hilden, Germany). HeLa human cervix carcinoma cells were obtained from RIKEN Cell Bank (Tsukuba, Japan). Dulbecco’s modified Eagle medium (D’MEM) and fetal bovine serum (FBS) were purchased from Invitrogen Corp. (Carlsbad, CA, USA). All other chemicals used were commercially available reagent-grade products.

\section{Construction of pDNA encoding MTS fused GFP}

To observe the intracellular trafficking of MTS fused GFP (MTS-GFP) in living cells, we constructed an MTS-GFP expression vector (pTENMG), as shown in Figure S1. cDNA encoding MTS derived from rat succinyl coA synthetase was amplified from rat liver cDNA by the polymerase chain reaction with high-fidelity Pyrobest DNA polymerase (Takara, Otsu, Japan). The amplified cDNA was inserted into pTriEx3 Neo Vector between the EcoR I and Nco I sites to generate pTENM. The GFP gene (Bgl II - EcoR I fragment) of the pTENG was then inserted into pTENM that had been pretreated with the same restriction enzymes (pTENMG). 


\section{DNA transfection and Intracellular observation of MTS fused GFP using confocal laser scanning}

\section{microscopy}

DNA transfection was carried out with the Lipofectamine Plus Reagent (Invitrogen) essentially according to the supplier's instructions. HeLa cells $\left(4 \times 10^{4}\right.$ cells $/ \mathrm{ml}, 3.5 \mathrm{~cm}$ dish) were cultured in D'MEM with $10 \%$ FBS under $5 \% \mathrm{CO} 2 /$ air at $37^{\circ} \mathrm{C}$ for $24 \mathrm{~h}$. After the serum free medium had been replaced, $1 \mu \mathrm{g}$ of pTENMG and pTENG plasmids containing the MTS-GFP and GFP genes were mixed with the reagents and transfected into the cells. After a 3-hr incubation under $5 \% \mathrm{CO}_{2}$ at $37^{\circ} \mathrm{C}$, the cells were further incubated in medium with $10 \%$ serum for $21 \mathrm{hr}$, and then washed with ice-cold PBS (-).The cells were excited by $488 \mathrm{~nm}$ light from an $\mathrm{Ar} / \mathrm{Kr}$ laser. A series of images were obtained by confocal laser scanning microscopy (LSM510; Carl Zeiss Co., Ltd., Jena, Germany) using a water immersion objective lens (Achroplan 63/NA 0.95) and a dichroic mirror (HFT488). The fluorescence detection channels were set to the following filters: BP 505-550 (green).

\section{Isolation of mitochondria from rat liver}

Mitochondria were isolated from livers obtained from adult male Wistar rats (6-8 weeks of age), essentially as described previously(Shinohara et al., 2002; Yamada et al., 2008). Rats were sacrificed and the livers removed after the bleeding had largely subsided, and then placed in approximately $20 \mathrm{~mL}$ of ice-cold mitochondrial isolation buffer containing EDTA [MIB (+): 250mM sucrose, 2 mMTris-HCl, 1 mM EDTA, pH 7.4] per $10 \mathrm{~g}$ of liver. All subsequent steps were carried out on ice. The livers were 
chopped into small pieces and the suspension homogenized in a glass homogenizer (50 mL capacity) with a pestle. Three complete up and down cycles with the pestle were made. The pestle was motor-driven and operated at approximately $550 \mathrm{rpm}$. The homogenate was diluted approximately 1:3 with MIB $(+)$ and centrifuged at $800 \mathrm{~g}$ for $5 \mathrm{~min}$. The supernatant was transferred into ice-cold tubes and centrifuged at 7,500g for $10 \mathrm{~min}$. The pellets were washed twice with MIB (+), and then once with EDTA-free MIB. Concentrations of mitochondrial proteins were determined using a BCA protein assay kit. All animal protocols were approved by the institutional animal care and research advisory committee at the Faculty of Pharmaceutical Sciences, Hokkaido University, Sapporo, Japan.

\section{Preparation of homogenate solution from HeLa cells}

HeLa cells were cultured in D'MEM, containing $10 \% \mathrm{FBS}$, under $5 \% \mathrm{CO}_{2} /$ air at $37^{\circ} \mathrm{C}$. A homogenate solution was obtained from a total of $5.0 \times 10^{6}$ cultured HeLa. The cells were washed with

ice-cold phosphate-buffered saline (PBS (-)), resuspended in MIB, and subjected to 20 strokes in a Dounce homogenizer on ice, to give a homogenate. 


\section{Supplementary Figure}

\section{Construction of pTENMG encoding MTS fused GFP (Figure S1)}

To observe the intracellular trafficking of MTS fused GFP (MTS-GFP) in living cells, we constructed an MTS-GFP expression vector (pTENMG) as shown in Figure S1.

Figure S1. Construction of pTENM and pTENMG.

A

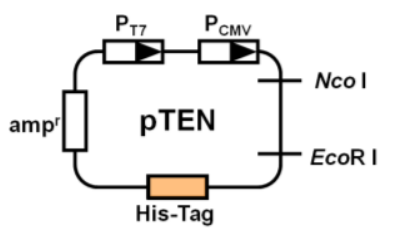

$$
\text { cDNA }
$$
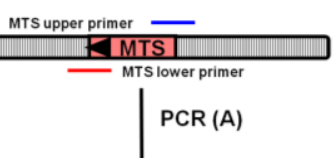

EcoR I Nco

Lcol I, EcoR I digestion (B)

Ligation (C)

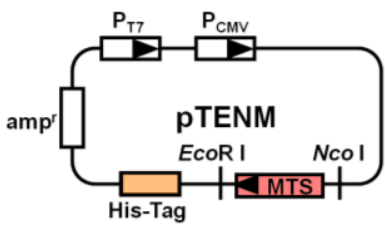

B
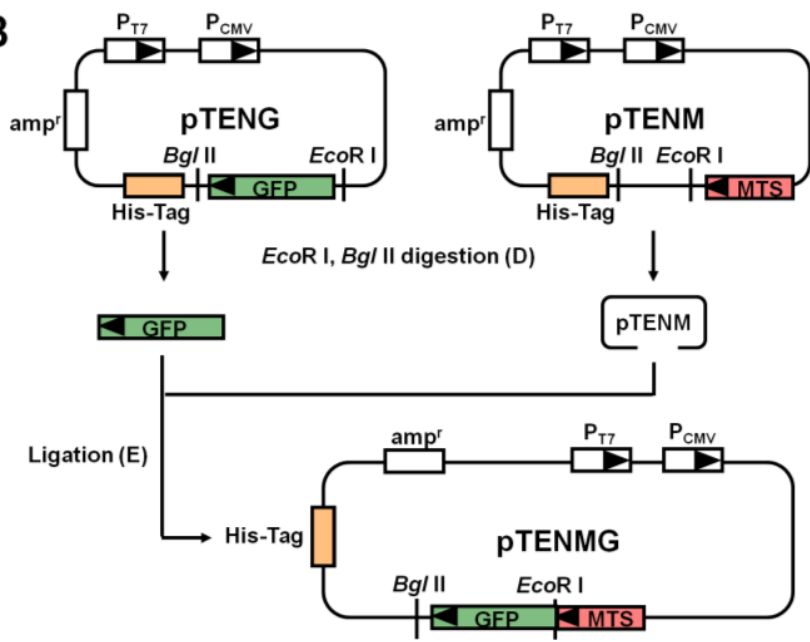

(A) Construction scheme of pTENM coding MTS. cDNA encoding MTS derived from rat succinyl coA synthetase was amplified from rat liver cDNA by the polymerase chain reaction with high-fidelity Pyrobest DNA polymerase. The amplified cDNA was inserted into pTriEx3 Neo Vector between the EcoR I and Nco I sites.

(B) Construction scheme of pTENMG coding MTS-GFP. The GFP gene (Bgl II - EcoR I fragment) of the pTENG coding GFP was inserted into pTENM pretreated with the same restriction enzymes. 
The characteristics of the MTS peptide are shown in Figure S2.

Figure S2. Characteristics of the MTS-Peptides
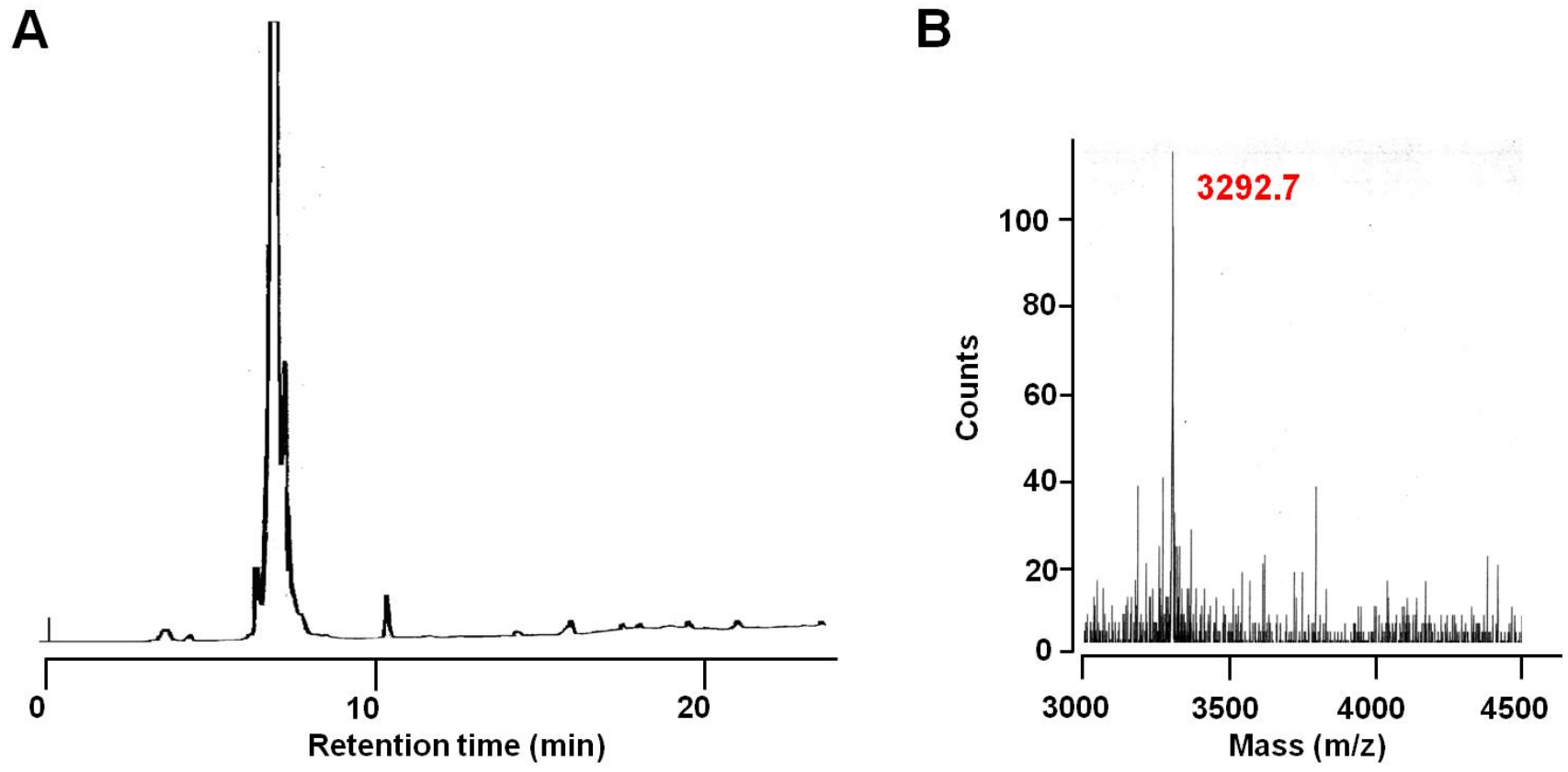

(A) Behavior upon reverse-phased HPLC for the reaction mixture of MTS peptide detected at $280 \mathrm{~nm}$.

(B) Molecular weight analysis of MTS peptide by MALDI-TOF-MS. The calculated molecular weight of MTS peptide is 3292.7 . 
Mitochondrial binding activities of R8-modified lipsomes (EPC/Chol=9:2) are shown in Figure S3. Mitochondrial binding activities were evaluated using isolated mitochondria.

Figure S3. Mitochondrial binding activity of the R8-modified lipsomes (EPC/Chol=9:2)

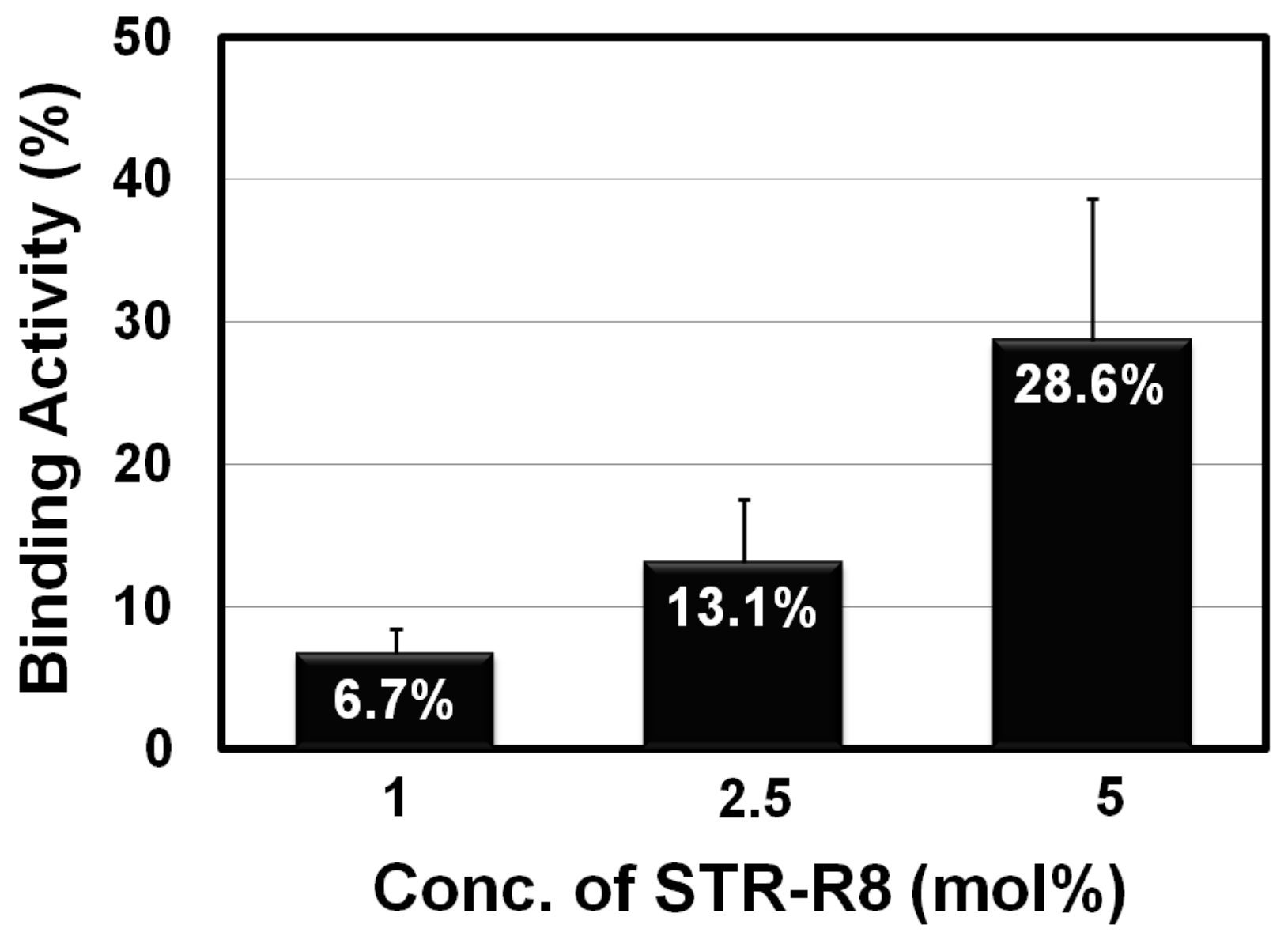

Mitochondrial binding activity of liposomes (EPC/Chol=9:2) conjugated with R8-peptide at various molar ratio. Data are means \pm S.D. $(n=3-5)$. 


\section{Fluorescent spectra to evaluate membrane fusion by FRET (Figure S4)}

We investigated the mitochondrial membrane fusion of carriers using FRET as described

previously (Maier et al., 2002; Struck et al., 1981; Yamada et al., 2008). Figure S4A shows the experimental protocol. Liposomal membranes were labeled with both NBD-DOPE (excitation at $460 \mathrm{~nm}$ and emission at $534 \mathrm{~nm}$ ) and rhodamine-DOPE (excitation at $550 \mathrm{~nm}$ and emission at $590 \mathrm{~nm}$ ) so that energy transfer will occur from NBD to rhodamine. Membrane fusion between the labeled liposomes and the mitochondria will lead to the diffusion of NBD and rhodamine into the lipid membranes, which causes a reduction in energy transfer, resulting in an increase in fluorescence intensity at $530 \mathrm{~nm}$. The maximum fluorescence was defined as the fluorescence of the liposomes when dissolved in detergent (Triton X-100). As shown in Figure S4B, the fluorescence intensity at $530 \mathrm{~nm}$ increased in the case of the MTS-modified MITO-Porter (Figure S4B, a)) and the R8-modified MITO-Porter (Figure S4B, b)), indicating a reduction in energy transfer due to fusion between the liposomal and mitochondrial membranes. On the other hand, no such increase at $530 \mathrm{~nm}$ was detected in the case of non-modified liposomes (Figure S4B, c)). 
Figure S4. The experimental protocol of membrane fusion analysis by FRET and fluorescent spectra to evaluate membrane fusion
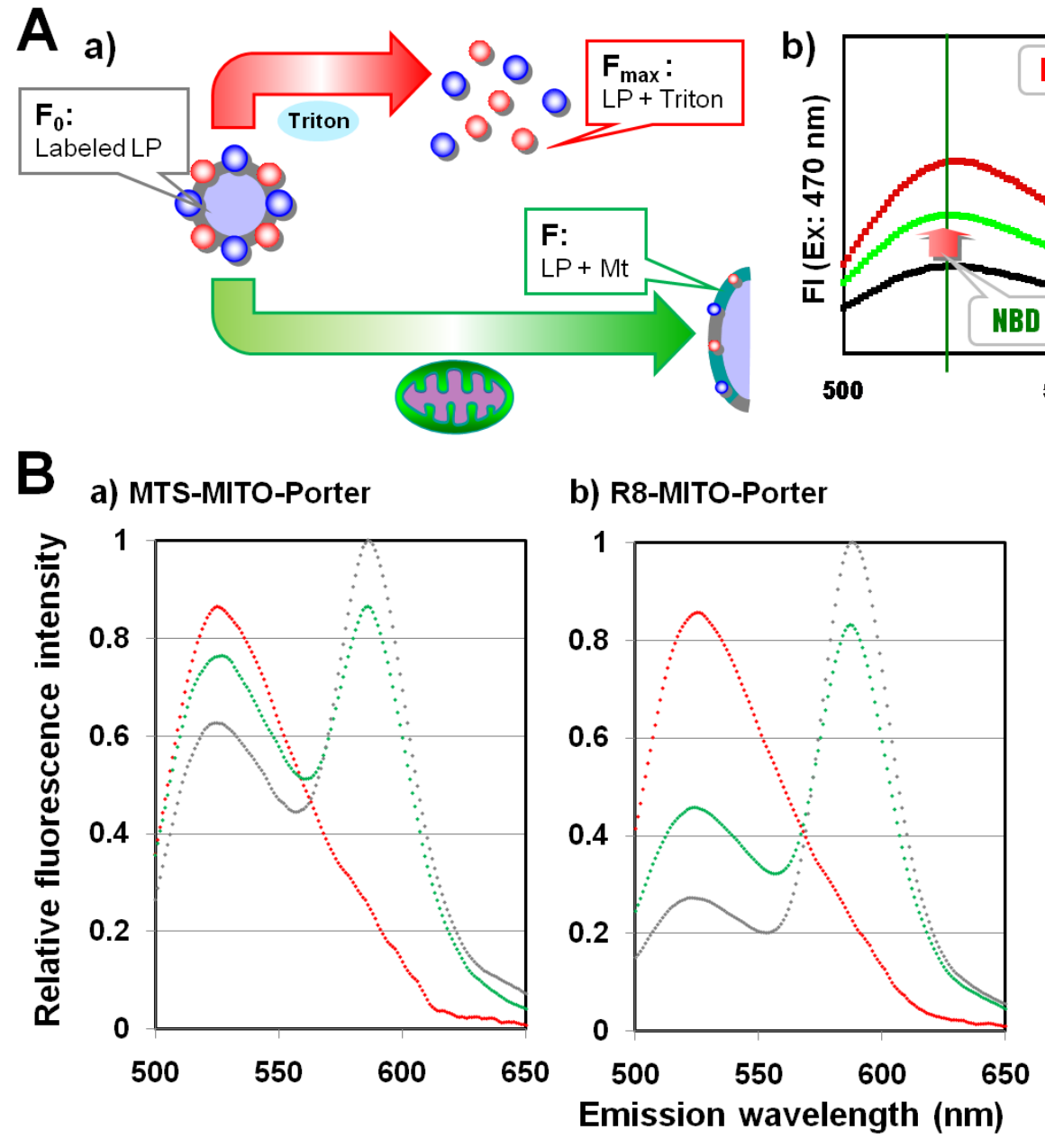

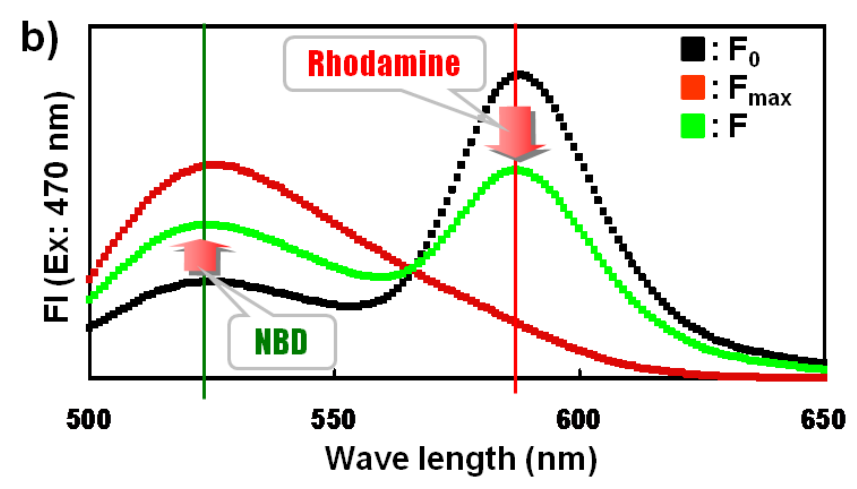

c) DOPE-LP

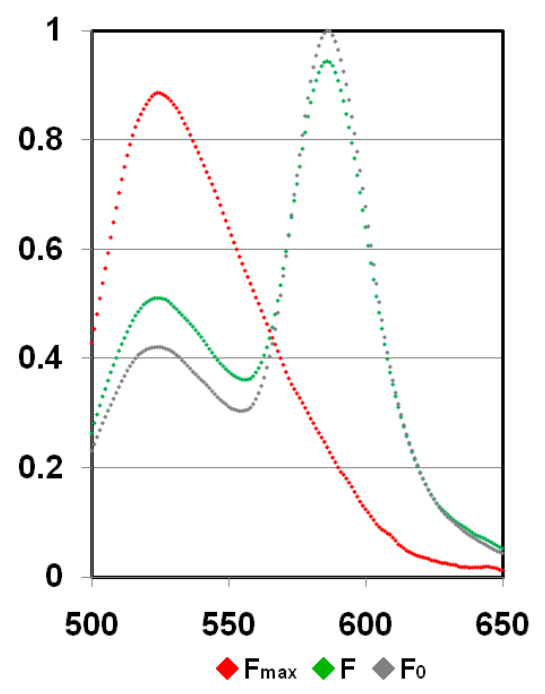

(A) The experimental protocol of the membrane fusion analysis using FRET. a) The experimental procedure was shown in Figure S4A. LP, liposomes; Mt, mitochondrion. b) The model fluorescent spectra was indicated in the case of $\mathrm{F}_{0}, \mathrm{~F}_{\max }$ and $\mathrm{F}$. Liposomal membranes were labeled with both NBD and rhodamine so that energy transfer will occur from NBD to rhodamine. Membrane fusion between the labeled liposomes and the mitochondria will lead to the diffusion of NBD and rhodamine into the lipid membranes, which causes a reduction in energy transfer. 
(B) Fluorescence spectra of a), b) and c) were observed in the case of excitation at $488 \mathrm{~nm}$ to evaluate membrane fusion by FRET. Red, green and gray lines indicate the fluorescence spectra of probe liposomes treated with triton, isolated mitochondria and buffer, respectively. 


\section{Supplementary Table}

Diameters of R8 modified liposomes composed of EPC and Chol (Table S1)

The diameters of the R8 modified liposomes composed of EPC/Chol (9:2) are summarized in Table S1.

Table S1. Diameters of R8 modified liposomes composed of EPC and Chol

\begin{tabular}{|c|c|c|c|}
\hline \multirow{2}{*}{} & \multicolumn{3}{|c|}{ Conc. of STR-R8 (mol\%) } \\
\cline { 2 - 4 } & 1 & 2.5 & 5 \\
\hline EPC/Chol (9:2) & $250 \pm 28$ & $159 \pm 23$ & $191 \pm 71$ \\
\hline
\end{tabular}

Data are means \pm S.D. $(n=3-5)$ 


\section{References}

Maier, O., Oberle, V., Hoekstra, D., 2002. Fluorescent lipid probes: some properties and applications (a review). Chem Phys Lipids 116, 3-18.

Mohanty, C., Das, M., Kanwar, J.R., Sahoo, S.K., 2011. Receptor mediated tumor targeting: an emerging approach for cancer therapy. Curr Drug Deliv 8, 45-58.

Shinohara, Y., Almofti, M.R., Yamamoto, T., Ishida, T., Kita, F., Kanzaki, H., Ohnishi, M., Yamashita, K., Shimizu, S., Terada, H., 2002. Permeability transition-independent release of mitochondrial cytochrome c induced by valinomycin. Eur J Biochem 269, 5224-5230.

Struck, D.K., Hoekstra, D., Pagano, R.E., 1981. Use of resonance energy transfer to monitor membrane fusion. Biochemistry 20, 4093-4099.

Yamada, Y., Akita, H., Kamiya, H., Kogure, K., Yamamoto, T., Shinohara, Y., Yamashita, K., Kobayashi, H., Kikuchi, H., Harashima, H., 2008. MITO-Porter: A liposome-based carrier system for delivery of macromolecules into mitochondria via membrane fusion. Biochimica et biophysica acta 1778, 423-432. 